\title{
Análisis Comparativo desde la Caracterización Curricular de Veintiséis Programas Presenciales de Maestrías en Administración en Colombia
}

\author{
Luis Botero, Ana M. Vélez-Evans y Pablo A. Múnera \\ Universidad Pontificia Bolivariana, Circular 1 \#70-01, Bloque 6, Segundo Piso, Medellín-Colombia \\ (e-mail: luishoracio.botero@upb.edu.co; ana.velez@upb.edu.co; pablo.munera@comunicaccion.com.co)
}

Recibido Mar. 5, 2019; Aceptado Abr. 23, 2019; Versión final May. 23, 2019, Publicado Oct. 2019

\begin{abstract}
Resumen
El artículo muestra los resultados de un estudio comparativo desde la caracterización curricular de veintiséis programas de Maestrías en Administración en Colombia, con el fin de servir de insumo en los procesos de construcción y renovación curricular o creación de nuevos programas. La metodología responde a una investigación descriptiva, con enfoque cualitativo. El estudio arrojó que estos programas son débiles en fundamentos epistemológicos, aunque en sus propuestas curriculares se evidencien seis áreas de formación y gestión, propias del dominio de las ciencias de la Administración. Se concluye que estos programas no muestran cambios curriculares sustanciales y que presenten factores diferenciadores en su oferta educativa.
\end{abstract}

Palabras clave: administración; posgrados; plan de estudios; mercado; gestión

\section{Comparative Analysis from the Curricular Characterization of twenty-six Master's Programs in Administration in Colombia}

\begin{abstract}
The article shows the results of a comparative study from the curricular characterization of twenty-six Master's programs in Administration in Colombia, with the objective of serving as an input in processes of construction and curricular renewal or creation of new programs. The methodology responds to a descriptive investigation, with a qualitative approach. The study showed that these programs are weak in epistemological foundations, although in their curricular proposals there are six areas of training and management, commonly found in the domain of Administration Sciences. It is concluded that these programs do not show substantial curricular changes and that present differentiating factors in their educational offer.
\end{abstract}

Keywords: administration; graduate studies; curriculum; market; management 


\section{INTRODUCCIÓN}

La formación de posgrados se ha convertido en un factor relevante y diferenciador en el ámbito de la educación superior de los países, dado que permite elevar, entre otras, la competitividad de las empresas (Akareem y Hossain, 2012). La demanda de estudiantes por esta oferta educativa está en auge en el mundo (Colombia no es la excepción). Al decir de Colvin (2015), el crecimiento no es igual en todos los continentes y las universidades de Europa, Asia y algunas de Latinoamérica tardaron en unirse al movimiento de ofertar programas de posgrados y, en particular, aquellos que requieren las organizaciones. En Colombia, se han realizado una serie de reformas e intervenciones al sistema de educación superior desde la década de los años 80 , lo que generó que aumentara no solo el número de estudiantes, sino de instituciones, tanto públicas como privadas, que ofertan programas de posgrados en áreas de la gestión. De acuerdo con datos estadísticos del Ministerio de Educación Superior, el número de estudiantes de educación superior es de 2.4 millones (dato consolidado al 2017). El sistema Nacional de Información de Educación Superior (SNIES, 2017) muestra que existe un total de 12.193 programas de educación superior vigentes.

Colombia necesita programas de Maestría en Administración que presenten al medio egresados con amplia visión y profundo conocimiento del mundo empresarial, los negocios y del mercado; preparados para leer e interpretar las nuevas dinámicas del entorno competitivo; capacitados para la formulación de estrategias coherentes y exitosas en los procesos funcionales de la organización; y que soporten, direccionen y creen organizaciones con visión global, orientadas a aumentar sus niveles de productividad y competitividad. La dinámica y los desafíos que rigen hoy a las organizaciones, obligan a pensar en programas interdisciplinarios e interculturales que den respuesta y solución a los múltiples problemas que las afectan, haciendo que los cursos a impartir, fortalezcan un conjunto de competencias relacionadas con los dominios de las ciencias de la Administración que les posibiliten la aplicación y generación de conocimientos científicos y tecnológicos en el sector productivo, social y de servicios (Gekara y Snell, 2018). Las Maestrías en Administración han sido criticadas desde diferentes autores, principalmente, por las siguientes razones: 1) Registrar un excesivo énfasis en aspectos funcionales y de forma; 2) Fomentar la aplicación de enfoques analíticos que poco o nada tienen que ver con los problemas reales de las organizaciones; 3) Concentrar los currículos en paradigmas positivistas de pensamiento; 4) Proponer mallas curriculares disciplinares, por asignaturas, no integradas y muchas veces desarticuladas y desalineadas (Costigan y Brink, 2015) y 5) Priorizar en el dominio de técnicas que responden más a modas administrativas que a resolver problemas de la gestión (Mintzberg y Gosling, 2002).

El plan de estudios, en general, exige nuevas competencias y habilidades, máxime donde el conocimiento se ha convertido en el elemento central del nuevo paradigma productivo y la transformación educativa pasa a ser un factor fundamental para el desarrollo de la innovación y la creatividad (Punie, 2007 y Salas, 2013). El énfasis de un programa de Maestría en Administración debe estar no sólo en la formación del capital humano, sino también en la gestión del nuevo conocimiento y de la innovación como fuentes de crecimiento económico sostenible (Ananiadou y Claro, 2009). En el siglo XXI, la sociedad del conocimiento invita a realizar cambios que incorporen la ciencia y tecnología como fuerzas productivas que permitan el desarrollo de los países, haciendo que éstos se concentren en la generación de capacidades investigativas e innovadoras en la educación superior (Gómez, Aranda y Santos, 2017). En el marco de lo expuesto, el objetivo de esta investigación es realizar un estudio comparativo desde la caracterización curricular de veintiséis (26) Maestrías en Administración ofertados por distintas universidades colombianas, con el fin de que sirva de insumo en los procesos de construcción y renovación curricular o creación de nuevos programas. Este artículo se centra en resolver la siguiente pregunta orientadora: ¿cuáles son las características curriculares existentes y diferenciadoras en los planes de estudio de las Maestrías en Administración en Colombia? La investigación se realizó en el año 2018 por docentes-investigadores pertenecientes a la Universidad Pontificia Bolivariana, sede Medellín, y recoge los resultados del proyecto: "Las maestrías en occidente: caracterización y análisis desde la perspectiva y para la prospectiva de universidad". El tipo de investigación es descriptiva, con enfoque cualitativo.

La relevancia de este estudio radica en que, dados los crecientes cuestionamientos que hay a las Maestrías en Administración, una forma de contribuir a su pertinencia, sobre todo, de tipo social, es través de una investigación descriptiva, con enfoque cualitativo que, consecuentemente, sirva para la construcción, a futuro, de las mallas curriculares de estos programas. Este artículo se constituye en un aporte, para que los administradores educativos revisen los planes de estudios ofertados, su composición en términos de créditos, duración, innovación e internacionalización. En Colombia, la formación de alto nivel (maestrías y doctorados) es una necesidad sentida para la comunidad académica, asunto que no es ajeno a las escuelas de Administración y Negocios, cuya necesidad de capacitación posgradual, más que necesaria, es imperativa, ya que se cuenta con una demanda suficiente, al menos así lo demuestra la gran cantidad de instituciones de educación superior que las ofertan. 


\section{TIPOLOGÍAS DE LAS MAESTRÍAS EN ADMINISTRACIÓN}

En el contexto mundial, la oferta de programas de Maestría en Administración es variada y responde a diferentes escuelas y tendencias de pensamiento, así como a multiplicidad de clasificaciones. Las tipologías responden a un modelo que en el núcleo básico de asignaturas no cambia. Muñoz (2004) indica que hay una corriente que pretende homogenizar con escasos matices que se pueden reducir a tres: 1) MBA prototipo; 2) Maestrías en Ciencias que siguen el paradigma epistemológico positivista de las ciencias duras y 3) Maestrías en Ciencias que enfatizan, desde lo epistemológico, en un paradigma hermenéutico más cercano con las ciencias sociales.

Para este estudio, y dada la oferta analizada, las Maestrías en Administración se clasifican en dos tipos: 1) De profundización que, según el decreto 1295 del 20 de abril de 2010 del Ministerio de Educación de Colombia, busca el desarrollo avanzado de competencias que permitan la solución de problemas o el análisis de situaciones particulares de carácter disciplinar, interdisciplinario o por medio de la asimilación o apropiación de saberes, metodologías y, según el caso, desarrollos científicos, tecnológicos o artísticos y pretenden dar respuesta, principalmente, a los intereses de las grandes empresas y son de carácter teórico-práctico, y 2) Investigativas, propias para la formación de investigadores que se preocupan por indagar y cuestionar los dominios de las ciencias de la Administración. Entre ambas, la diferencia marcada consiste en que las segundas dan mayor rigor y énfasis a la investigación en sus diferentes modalidades del trabajo de grado y tienen una postura crítica frente a los dominios de su área de conocimiento. En este asunto, incluso, se afirma que no está sellado el debate sobre el objeto de estudio de las ciencias de la Administración y que, por ello, es necesario el plantear, desde lo epistemológico, un objeto de estudio que dé cuenta de la complejidad misma de las organizaciones y de las interacciones humanas.

Dada la no univocidad del debate epistemológico, existen otras tipologías para las Maestrías en Administración que las clasifica en dos grandes vertientes: 1). Practicistas y 2) Intelectualistas. Y podríamos señalar, una más que corresponde a aquellas practicistas que privilegian el aprender-haciendo. Muñoz (2004) indica que este tipo de programas se diseña en torno al eje fundamentación humanista-contextualizacióninstrumentación genérica, actitudinal y especializada. No obstante, lo más frecuente es que se obvie lo humanístico y se concentre en lo instrumental, quedando reducida la formación a aspectos que privilegian el hacer cómo y, a lo sumo, con algo de contextualización. Los estudiantes de este tipo de programas de maestrías suelen buscar respuestas prácticas a los problemas de la gestión y, por ello, demandan ofertas que traten de dar respuesta al quehacer y al cómo de la gestión y no a los qué y por qué de la misma. Por su parte, las maestrías científicas con orientación cuantitativa se diseñan alrededor del eje fundamentacióncontextualización-especialización (opcional)-investigación. Y en la que tiene orientación cualitativahumanística, se articulan básicamente así: fundamentación-especialización (opcional)-investigación.

En la Tabla 1 se muestra una síntesis y comparación de los tipos básicos de Maestrías en Administración, tipo MBA y afines y Maestría en Ciencias de corte positivista y de corte hermenéutico.

\section{METODOLOGÍA}

La investigación en cuestión es descriptiva, con enfoque cualitativo. Permite estudiar un fenómeno, a través del entendimiento de sus atributos (caracterización curricular) y, a partir de información documental, se da cuenta de la manera en la cual se encuentra la estructura curricular y el análisis de dominio de las ciencias de la Administración. De manera puntual, para la realización del estudio comparativo, se consideraron las mallas curriculares, consultadas en octubre de 2018, a través de los portales institucionales de las diferentes universidades estudiadas. Se consideraron aspectos curriculares (básicamente mesocurriculares) de veintiséis (26) Maestrías en Administración de un total de cincuenta (50) que ofertan este tipo de programas (SNIES, 2017), pertenecientes al sector público y privado, bajo la modalidad presencial en diferentes departamentos de Colombia y se tuvo en cuenta, de manera puntual, un análisis respecto del plan de estudios. La evidencia concreta de las denominaciones de las asignaturas y su valor en créditos, permitió hacer las inferencias con relación al dominio de las ciencias de la Administración. Se definieron seis (6) áreas académicas como criterios de clasificación, las cuales se entienden como espacios demarcados que reúnen conocimientos organizados dentro de un componente y que cumplen unos propósitos formativos específicos (áreas inmersas en el análisis de dominio de las ciencias de la Administración). Estas áreas dan cuenta de los asuntos propios de la epistemología de la administración; de las áreas funcionales básicas de las organizaciones y del estudio comparativo realizado a las asignaturas transversales y recurrentes en la mayoría de las mallas curriculares de las instituciones, objeto de estudio. Dichas áreas son: 1). Dirección y Gerencia; 2). Operaciones y Marketing; 3). Economía de Empresas y Finanzas; 4) Innovación; 5) Investigación y 6). Ética. Para completar el análisis, se examinaron igualmente los cursos electivos y optativos. 


\begin{tabular}{|c|c|c|c|c|c|c|c|}
\hline 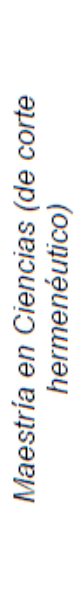 & \multirow[b]{2}{*}{ 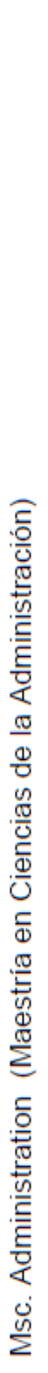 } & \multirow{2}{*}{ 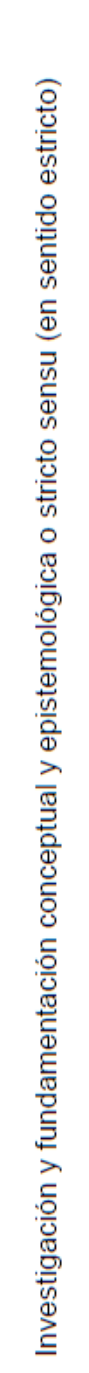 } & \multirow{2}{*}{ 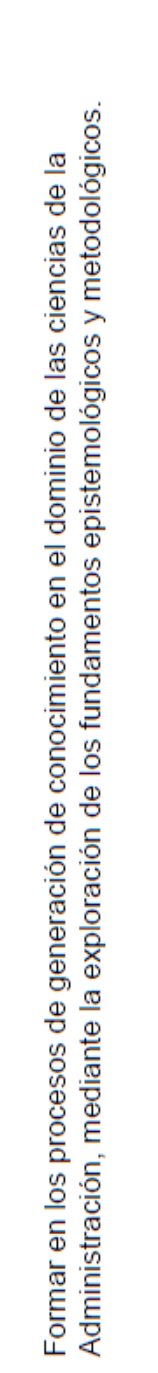 } & 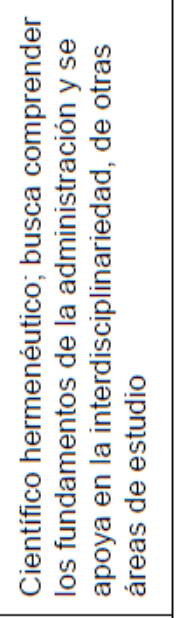 & \multirow{2}{*}{ 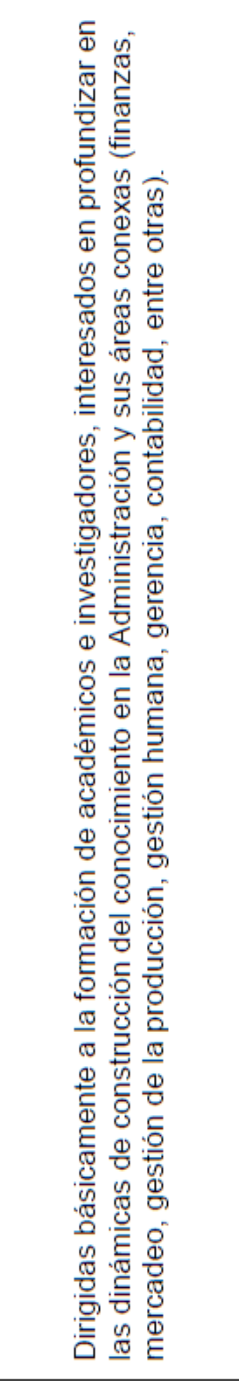 } & \multicolumn{2}{|c|}{ 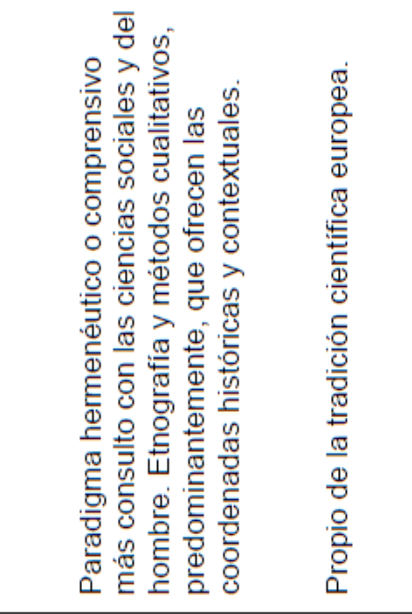 } \\
\hline 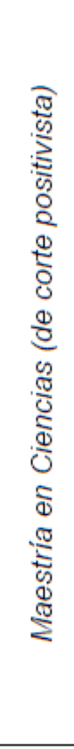 & & & & 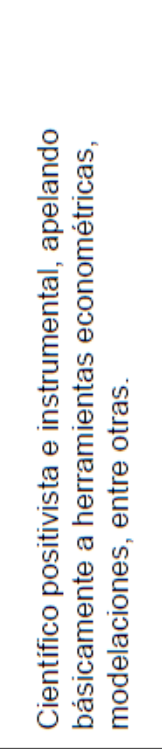 & & 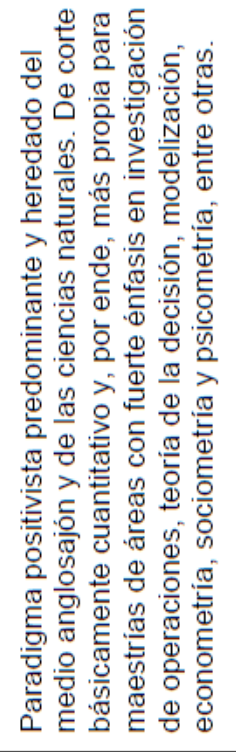 & 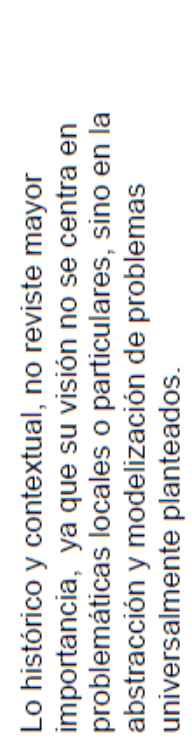 \\
\hline 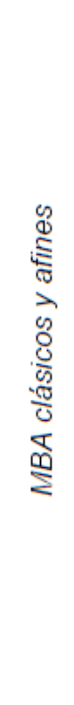 & 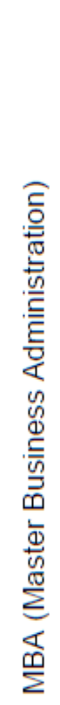 & 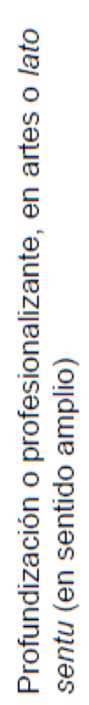 & 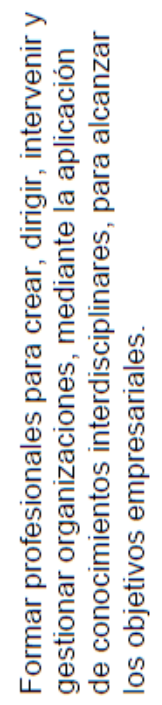 & 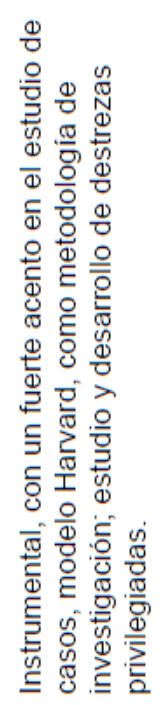 & 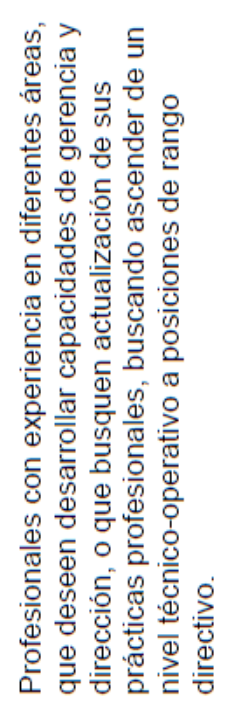 & 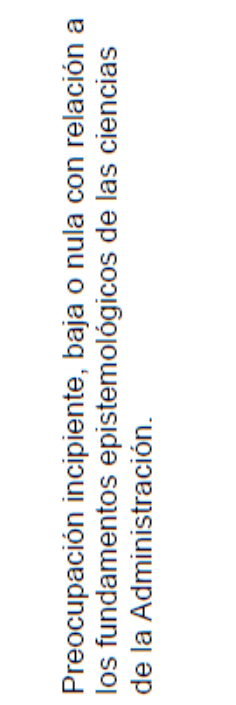 & 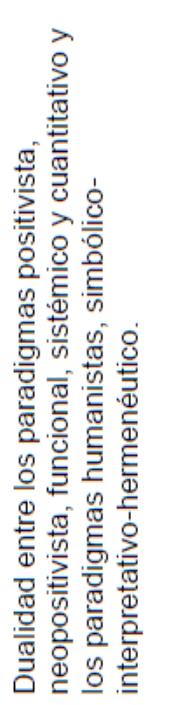 \\
\hline & $\vec{F}$ & 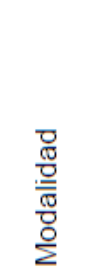 & 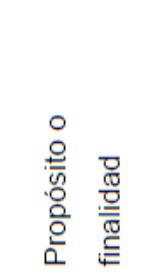 & 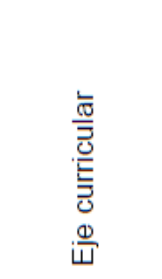 & 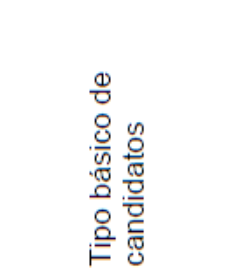 & 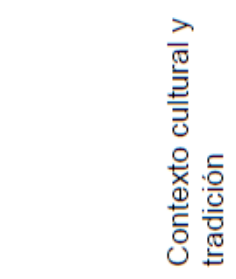 & \\
\hline
\end{tabular}




\section{RESULTADOS}

Los resultados o hallazgos fueron subdivididos en dos: 1) Plan de estudios y 2) Innovación y currículo, y se explican a continuación:

\section{Plan de estudios}

El plan de estudios, se entiende como un esquema estructurado, de ciclos, áreas y cursos obligatorios, electivos y optativos. Este plan debe establecer los propósitos de formación por ciclos, áreas y cursos; la metodología de trabajo; la distribución del tiempo y los criterios de evaluación; la administración y gestión del currículo. Entre tanto, la malla curricular es la representación gráfica de la distribución de los ciclos de formación y de los cursos contemplados en el plan de estudios; esta permite hacer visibles las relaciones de prioridad, secuencialización y articulación de los cursos entre ellos y con los ciclos. En promedio, el plan de estudios de los programas analizados tiene cincuenta y dos (52) créditos académicos, donde un crédito corresponde a una medida equivalente al tiempo de trabajo de los estudiantes, para alcanzar las metas de aprendizaje; diecisiete (17) asignaturas a cursar; dieciséis (16) créditos por semestre académico y una duración de dos (2) años. La mayor cantidad de créditos académicos de las Maestrías en Administración analizadas se otorgan al área de Dirección y Gerencia (40\%); seguida de los cursos propios de formación y desarrollo investigativo (16\%); Economía de Empresas y Finanzas (12\%); Operaciones y Marketing (11\%), y cursos electivos (10\%). Las menores asignaciones de créditos de las áreas de estudio recurrentes corresponden al área de Innovación (8\%) y Ética (2\%). En la Tabla 2 se muestra las áreas seleccionadas para el análisis, propias del dominio de las ciencias de la Administración. La primera corresponde a Dirección y Gerencia, con la mayor cantidad de créditos asignados (40\%), lo que evidencia la importancia que el plan de estudios le da al eje de formación en Administración; sin embargo, esta formación es descriptiva y prescriptiva, dado que privilegia componentes curriculares que responden a asuntos coyunturales y no aquellos propios de estos dominios (Alonderiene, y Majauskaite, 2016).

Tabla 2: Áreas de análisis del dominio de las ciencias de la Administración

\begin{tabular}{|l|c|c|c|c|c|c|c|}
\cline { 2 - 8 } \multicolumn{1}{c|}{} & $\begin{array}{c}\text { Dirección y } \\
\text { Gerencia }\end{array}$ & $\begin{array}{c}\text { Operaciones } \\
\text { y marketing }\end{array}$ & $\begin{array}{c}\text { Economía de } \\
\text { empresas y } \\
\text { finanzas }\end{array}$ & Innovación & Investigación & Ética & Electivas \\
\hline Total Créditos & 541 & 153 & 165 & 109 & 213 & 32 & 142 \\
\hline Promedio Cursos Área & 7 & 2 & 2 & 1 & 3 & 0 & 2 \\
\hline Porcentaje en créditos & $40 \%$ & $11 \%$ & $12 \%$ & $8 \%$ & $16 \%$ & $2 \%$ & $10 \%$ \\
\hline
\end{tabular}

Aunque el plan de estudios de las maestrías analizadas corresponde a un programa de profundización y no de investigación, este último equivale a un dieciséis por ciento (16\%) de asignación de créditos, donde se pretende que el estudiante aplique competencias básicas para la investigación. Si bien el porcentaje de participación es el segundo de las seis áreas seleccionadas, basados en experiencia empírica, se registra un nivel de desarrollo insuficiente en competencias investigativas que brinden herramientas sobre aspectos propios del diseño metodológico, los instrumentos de recolección de la información y la gestión de la divulgación del conocimiento.

Los cursos del área de Economía de Empresas y Finanzas (12\%), comprenden los conceptos principales sobre la gestión de los recursos financieros, el papel de la organización en la economía de mercado y el rol del directivo como agente económico. El área de Operaciones y Marketing queda en un cuarto lugar con un once por ciento (11\%) de los créditos asignados con asignaturas que abordan los conceptos relacionados con la gestión de la operación de las organizaciones, la logística de los procesos, el marketing de los productos y servicios. Se presenta un componente flexible de las Maestrías en Administración, a través de los créditos asignados a cursos electivos $u$ optativos (10\%) con los cuales las universidades ofertan asignaturas complementarias a la formación, donde el estudiante adquiere conocimientos, habilidades y destrezas en aquellas áreas que sean de su especial interés. Las dos áreas con un menor porcentaje de asignación de créditos son Innovación y Ética. En la primera ( $8 \%$ de su valor en créditos) el estudiante identifica la cultura del emprendimiento e innovación, determina los principales retos que enfrenta un emprendedor e innovador, orienta la innovación tecnológica como una de las principales fuentes de construcción de ventajas competitivas de largo plazo para la empresa. Sin embargo, es menester señalar que esta área y sus asignaturas son parte de las modificaciones recientes al plan de estudios de estos programas, situación que implica que no existan evidencias concretas del impacto real en el medio. Y, en la segunda, aunque los programas tienen módulos sobre el tema, solo equivale al $2 \%$ del valor de los créditos; por lo tanto, debe hacer una apuesta en el plan de estudios por incrementar el valor en créditos, dado que es de gran importancia en la formación de los magister que serán los futuros líderes empresariales y quienes deberán incorporar la ética en cada decisión que tomen en los negocios. El área se encuentra en función del respeto a la vida, a los 
valores morales y sociales y hacia los demás y al cuidado del medio ambiente. En la Tabla 3 se muestra los veintiséis (26) programas de Maestrías en Administración, modalidad presencial, y su valor en créditos; de esta, se registran cinco (5) universidades con más de sesenta (60) créditos académicos, lo que equivale a $43.19 \%$; entre 50 y 59 créditos, once (11), cuyo porcentaje es $42,3 \%$ y menos de 49 créditos y hasta 39 , diez (10) instituciones, lo que equivale al $38.46 \%$.

Tabla 3: Maestrías en Administración, presencial y su valor en créditos

\begin{tabular}{|c|c|c|c|}
\hline Universidad & Sector & $\begin{array}{c}\text { Ubicación } \\
\text { Departamento/ } \\
\text { Ciudad }\end{array}$ & Créditos \\
\hline 1. Eafit (MBA Global) & Privado & $\begin{array}{l}\text { Antioquia - } \\
\text { Medellín }\end{array}$ & 67 \\
\hline 2. Externado de Colombia & Privado & $\begin{array}{l}\text { Cundinamarca - } \\
\text { Bogotá }\end{array}$ & 65 \\
\hline $\begin{array}{l}\text { Colegio de Estudios Superiores de Administración- } \\
\text { Cesa- (MBA Internacional) }\end{array}$ & Privado & $\begin{array}{c}\text { Cundinamarca - } \\
\text { Bogotá }\end{array}$ & 64 \\
\hline 4. De La Sabana & Privado & $\begin{array}{l}\text { Cundinamarca - } \\
\text { Chía }\end{array}$ & 62 \\
\hline 5. Del Norte & Privado & $\begin{array}{l}\text { Atlántico - } \\
\text { Barranquilla }\end{array}$ & 60 \\
\hline 6. De Los Andes & Privado & $\begin{array}{l}\text { Cundinamarca - } \\
\text { Bogotá }\end{array}$ & 59 \\
\hline 7. Universitaria de Investigación y Desarrollo - UDI- & Privado & $\begin{array}{c}\text { Santander - } \\
\text { Bucaramanga }\end{array}$ & 56 \\
\hline 8. Nacional de Colombia & Público & $\begin{array}{l}\text { Cundinamarca - } \\
\text { Bogotá }\end{array}$ & 56 \\
\hline 9. De La Salle & Privado & $\begin{array}{l}\text { Cundinamarca - } \\
\text { Bogotá }\end{array}$ & 55 \\
\hline $\begin{array}{l}\text { 10. Colegio de Estudios Superiores de Administración- } \\
\text { CESA- }\end{array}$ & Privado & $\begin{array}{c}\text { Cundinamarca - } \\
\text { Bogotá }\end{array}$ & 54 \\
\hline 11. Pontificia Universidad Javeriana & Privado & $\begin{array}{c}\text { Cundinamarca - } \\
\text { Bogotá }\end{array}$ & 54 \\
\hline 12. Autónoma de Bucaramanga -UNAB- & Privado & $\begin{array}{l}\text { Santander - } \\
\text { Bucaramanga }\end{array}$ & 52 \\
\hline 13. Icesi & Privado & Valle - Cali & 52 \\
\hline 14. Católica Luis Amigó-Funlam- & Privado & $\begin{array}{l}\text { Antioquia - } \\
\text { Medellín }\end{array}$ & 51 \\
\hline 15. De Antioquia & Público & $\begin{array}{l}\text { Antioquia - } \\
\text { Medellín }\end{array}$ & 51 \\
\hline 16. Pedagógica y Tecnológica de Colombia - UPTC- & Público & Boyacá - Tunja & 50 \\
\hline 17. Pontificia Universidad Javeriana (sede Cali) & Privado & Valle - Cali & 50 \\
\hline 18. Industrial de Santander & Público & $\begin{array}{c}\text { Santander - } \\
\text { Bucaramanga }\end{array}$ & 50 \\
\hline 19. Eafit & Privado & $\begin{array}{l}\text { Antioquia - } \\
\text { Medellín }\end{array}$ & 49 \\
\hline 20. De Medellín & Privado & $\begin{array}{l}\text { Antioquia - } \\
\text { Medellín }\end{array}$ & 48 \\
\hline 21. Autónoma de Occidente & Privado & Valle-Cali & 48 \\
\hline 22. Tecnológica de Bolívar & Privado & $\begin{array}{c}\text { Bolivar - } \\
\text { Cartagena } \\
\end{array}$ & 47 \\
\hline 23. Sergio Arboleda & Privado & $\begin{array}{c}\text { Cundinamarca - } \\
\text { Bogotá }\end{array}$ & 45 \\
\hline 24. Pontificia Bolivariana & Privado & $\begin{array}{l}\text { Antioquia - } \\
\text { Medellín }\end{array}$ & 44 \\
\hline 25. Colegio Mayor de Nuestra Señora del Rosario & Privado & $\begin{array}{l}\text { Cundinamarca - } \\
\text { Bogotá }\end{array}$ & 40 \\
\hline 26. Del Valle & Público & Valle - Cali & 39 \\
\hline
\end{tabular}

El valor total en créditos varía entre 39 y 67 , hecho que evidencia desviaciones frente a lo que corresponde a la media y a la no univocidad y exigencia por parte de las universidades que los ofertan y del Ministerio de Educación de Colombia que las vigila. Un crédito académico es la unidad de medida de trabajo del estudiante en tiempo en función de las competencias profesionales y académicas que se espera que el programa desarrolle., que en Colombia corresponde a 48 horas. El número de créditos de cada programa presenta una dispersión muy alta para un programa de Maestría en Administración, hasta el punto que el de mayor número de créditos (67) duplica al de menor (39), lo que constituye un factor problematizador en términos de exigencia académica y de igualdad de condiciones. El Decreto 2566 de Septiembre 10 de 2003 del Ministerio de Educación Nacional de Colombia no se determinan consideraciones acerca de la cantidad mínima o máxima 
de créditos que debe tener un programa de Maestría, estableciendo solo criterios de calidad en cuanto a la fundamentación teórica, práctica y metodológica del programa; principios y propósitos que orientan la formación; estructura y organización de los contenidos curriculares; estrategias para el trabajo interdisciplinario; modelo y estrategias pedagógicas y el perfil de formación.

Se deduce, una tendencia en estos programas a ser poco diferenciados, lo cual podría interpretarse, desde una perspectiva neutra, en que es un producto/servicio maduro que no deja mucho espacio para la diferenciación, situación que se contradice en parte con la cantidad de créditos de cada programa. Desde otra perspectiva, más crítica, podría leerse que los programas revisados responden a una especie de proforma, dando cuenta de poca reflexión y rigor académico en lo epistemológico y curricular. De hecho, se encuentran casos de maestrías que son casi idénticas unas a otras en su plan de estudios a pesar de pertenecer a universidades y regiones muy diferentes. Es necesario que exista mayor coherencia entre el diseño curricular y la pertinencia social de los programas de Maestría en Administración. Ello, implica que se busque que las seis áreas seleccionadas tengan un hilo conductor, donde cada módulo responda por un eje articuladororientador que dé respuesta a las necesidades del educando y a los retos que el entorno demanda del futuro egresado.

\section{Innovación y currículo}

Los procesos de innovación en las Escuelas de Negocios se han centrado en revisar y reestructurar el plan de estudios en un intento por atraer a los estudiantes, así como para actualizar los perfiles de egresados, competir con los requerimientos del mundo de los negocios y asegurar un nivel de permanencia en la oferta de procesos de formación en el nivel de posgrados. Tras revisar los veintiséis (26) programas de Maestría en Administración, la principal estrategia de innovación observada corresponde al diseño de mallas curriculares enfocadas en privilegiar más las técnicas que conocimientos; por tanto, son más de forma que de fondo; conceptos antes que desarrollar criterios propios del dominio de las ciencias de la Administración y a establecer dimensiones o campos disciplinares que, muchas veces, no conversan entre sí. Bajo el pretexto de la necesidad de ofertar programas innovadores, diseñan y ofertan cursos electivos u optativos, tales como emprendimiento e innovación o bien se apela a técnicas propias del "aprender, haciendo".

\section{DISCUSIÓN}

Las críticas de los estudiantes y egresados coinciden en que las universidades ofertan programas teóricos, donde abundan discursos prescriptivos que no van al ritmo de las exigencias del entorno, máxime en un cambio de época como la actual, donde se evidencian las exigencias de una Cuarta Revolución Industrial o Revolución 4.0 (Schwab, 2016). Sin embargo, y pese a las críticas, las Maestrías en Administración continúan en el top de la oferta de programas de posgrados, dado que mantienen la promesa de preparar sistemáticamente a sus graduados para sus funciones directivas. Hay posturas crecientes de que estos programas están perdiendo su relevancia, basándose en la evidencia empírica de que las competencias que los gerentes precisan son las que menos están representadas en las mallas curriculares (Tan y Ko, 2019). En la práctica, siguen siendo pieza central de la educación en gestión y conservan la promesa de preparar a los estudiantes, para sus funciones directivas. En este sentido, para los roles gerenciales debe considerarse no solo el plan de estudios, sino también el aprendizaje real y práctico (Tan y Ko, 2019). Existen estudios que señalan una marcada desalineación entre las competencias necesarias para la efectividad de gestión y las que se imparten en los cursos de las Maestrías en Administración (Costigan y Brink, 2015).

Los programas analizados olvidan la formación en habilidades suaves o blandas que corresponden a aquellas que no están relacionadas únicamente con el conocimiento, sino con la puesta en práctica de una combinación de habilidades interpersonales, entendidas estas últimas como aquellas que permiten tener una mejor comunicación con otras personas; habilidades sociales y de comunicación; aptitudes y capacidad de acercamiento con los demás y que son las que más necesitan aplicar los estudiantes y gerentes, tipo liderazgo, trabajo en equipo y comunicación, entre otras. Las habilidades suaves o blandas son fundamentales para el éxito de la gestión; sin embargo, en las mallas curriculares comparadas, estos saberes tienen un bajo componente porcentual e, incluso, en algunos casos son incorporados a otras asignaturas, de manera tangencial. Para la combinación de habilidades, en un trabajo investigativo de Beenen, Pichler, y Davoudpour (2018), se presenta una encuesta a 182 profesionales, aspirante a ser admitidos en programas, tipo MBA, y pertenecientes a 24 países, la cual reveló cómo se conceptualizaron las habilidades interpersonales y sugiere que las habilidades interpersonales y las sociales no son sinónimos. Los resultados también indicaron que solo el $30 \%$ de los programas de MBA de los Estados Unidos utilizan criterios específicos, para evaluar las habilidades interpersonales de los aspirantes, y el resto utiliza criterios no específicos o ningún método de evaluación. Además, y tal como lo afirma Ortega (2017), los componentes de uso de redes sociales y de interacciones digitales con propósitos académicos en ámbitos nacionales e internacionales y correspondientes al componente innovación y currículo en la sección de resultados, no se evidencian como 
prácticas de los docentes y estudiantes. Por su parte, Yáñez y Soria (2017) señalan que las prácticas docentes deben estar relacionadas con las metodologías que imparten los educadores, así como al dominio de las ciencias que estos representan. Lo anterior, coincide con el trabajo de Rodríguez e Hinojo (2017), quienes afirman que la docencia debe contar con un amplio conocimiento en pedagogía y en disciplinas relacionadas con la educación.

La investigación, si bien no es la esencia de las Maestrías en Administración en profundización, resulta preocupante los resultados que esta arroja. Del análisis, se determina que estos programas son precarios en materia de investigación; abundan los trabajos de monografías, estudios de caso o planes de empresas, incluso hasta ensayos. Los productos que de allí se derivan no son relevantes, máxime si consideramos las exigencias de visibilidad e impactos en ámbitos internacionales que precisa la investigación. Respecto a los aspectos atinentes a una educación con visión crítica, Ghoshal (2005) advierte que las Maestrías en Administración deberían forman gerentes con un sentido amplio de la responsabilidad ética y social, dando cabida a jugar un papel más propositivo en la sociedad. El plan de estudios debe articularse con los problemas y dominios reales de la administración y la gestión, mediante un ejercicio de intervención epistemológico y desde una apuesta real por entender y resolver los retos que aquejan a las ciencias de la Administración (Barro y Lee, 2013). Son múltiples y variadas las prácticas tomadas de las escuelas de negocios de los Estados Unidos, evidenciando la carencia en apuestas propias, ajustadas a las realidades locales, regionales o nacionales, así como posturas en favor de los dominios de las ciencias de la Administración. Para Ghoshal (2005), las Maestrías en Administración privilegian más aspectos de forma que de fondo; su preocupación se limita a lograr seguidores, no estudiantes críticos que aporten nuevo conocimiento. Asuntos como la transformación digital e, incluso, las temáticas propias de la Cuarta Revolución Industrial (Big data, Internet de las cosas, Inteligencia artificial, Blockchain, entre otras) son temas ausentes en las mallas curriculares analizadas.

Estudios como los de Olivencia, García y Galán (2014) establecen que el aprendizaje es un proceso dialógico entre docentes y estudiantes, de concepción socio-constructivista, situación que no se evidencia en las prácticas de las maestrías analizadas, dejando a un lado lo que estos autores destacan como prácticas docentes y de comunicación entre los actores, para promover la construcción del conocimiento. Es importante pensar en un proceso con diferentes líneas formativas que brinde mayores saberes y conocimientos ante los retos de un cambio de época como la actual. Estos programas precisan de una transformación curricular, lo que implica, necesariamente, también un cambio en las comunidades académicas y, sobre todo, en los docentes que imparten este tipo de formación. Los programas de Maestría en Administración deben formar a los líderes del mañana y es por eso que se necesitan profesores y gerentes de alto nivel que estén facultados, para asumir responsabilidades más amplias y estratégicas. La asignación de créditos fomenta la autonomía del estudiante, para elegir actividades formativas que responden, de modo directo, a sus intereses y motivaciones personales y la producción y el acceso a diferentes tipos de experiencias de aprendizaje flexible (Barth, Adoment, Fischer, Richter y Rieckmann, 2014) facilita una clara organización de los deberes del estudiante en los períodos académicos que fije la institución y ajusta el ritmo del proceso de formación a las diferencias individuales de los estudiantes.

Las disrupciones que se avecinan imponen desafíos a docentes y estudiantes y, en ello, no hay puntos intermedios, sino apuestas claras y contundentes, para asumir los cambios exponenciales en materia de procesos de enseñanza en pregrado y posgrados, de los cuales las Maestrías en Administración no están exentas. Al decir de Rubin y Dierdorff (2013), el futuro de los programas de Maestría en Administración, más allá de las críticas y de las recetas que estos suelen enseñar, la gestión del capital humano y la capacitación en competencias para la toma de decisiones deben tener mayor participación en las mallas curriculares y en las metas de aprendizaje. Un amplio plan de estudios, con temas multidisciplinarios y prácticos, pueden permanecer, para abordar, de forma parcial, los complejos retos que enfrentan los líderes empresariales (Gupta, Saunders y Smith, 2007).

\section{CONCLUSIONES}

Los resultados encontrados permiten obtener las siguientes conclusiones: 1) Los programas de Maestrías en Administración comparados, no muestran cambios curriculares sustanciales que presenten factores diferenciadores en su oferta educativa; algunos de ellos son casi idénticos en su malla curricular, evidenciando poca reflexión y rigor académico; 2) Se presenta una dispersión alta en la oferta cuantitativa en créditos académicos y dichos créditos responden más a una unidad de valoración para el cálculo del costo de la matrícula que a la finalidad de permitir autonomía en el proceso de aprendizaje del estudiante; 3) Los programas que oferten saberes más específicos que den respuesta a las necesidades reales del sector productivo, serán los que, a largo plazo, mayor demanda obtendrán por parte de los estudiantes y 4) Investigaciones futuras deben centrarse en indagar a empleadores y egresados por lo que estos requieren en cuanto a formación para la gestión. 


\section{REFERENCIAS}

Akareem, H. y S. Hossain, Perception of Education Quality in Private Universities of Bangladesh: A study from Students Perspective, doi: 10.1080/08841241.2012.705792, Journal of Marketing for Higher Education, 22(1), 11-33 (2012)

Alonderiene, R. y M. Majauskaite, Leadership Style and Job Satisfaction in Higher Education Institutions, doi: https://doi.org/10.1108/IJEM-08-2014-0106, International Journal of Educational Management, 30(1), 140-164 (2016)

Ananiadou, K. y M. Claro, 21ST Century Skills and Competences for New Millennium Learners in OECD Countries, doi: 10.1787/19939019, OECD Education Working Papers, 41, 1-34 (2009)

Barro, R.J., y J.W. Lee, A New Data Set of Educational Attainment in the World, 1950-2010, doi: https://doi.org/10.1016/j.jdeveco.2012.10.001, Journal of Development Economics, 104, 184-198 (2013)

Barth, M., M. Adoment y otros tres autores, Learning to Change Universities from within: a Service-Learning Perspective on Promoting Sustainable Consumption in Higher Education, doi: https://doi.org/10.1016/j.jclepro.2013.04.006, Journal of Cleaner Production, 62, 72-81 (2014)

Beenen, G., S Pichler, y S. Davoudpour, Interpersonal skills in MBA admissions: How are they conceptualized and assessed?, doi:10.1177/1052562917703743, Journal of Management Education, 42(1), 34-54 (2018)

Colvin, J. W., Peer Mentoring and Tutoring in Higher Education, doi: 10.1080/13611260601086345, Exploring Learning \& Teaching in Higher Education, 207-229 (2015)

Costigan, R.D., y K. Brink, Another perspective on MBA program alignment: An investigation of learning goals, doi:10.5465/amle.2013.0315, Academy of Management Learning and Education, 14(2), 260-276 (2015)

Gekara, V. y D. Snell, Designing and Delivering Skills Transferability and Employment Mobility: The Challenges of a Markent-Driven Vocational Education and Training System, doi: 10.1080/13636820.2017.1392996, Journal of Vocational Education \& Training, 70 (1), 107 - 129 (2018)

Ghoshal, S., Bad Management Theories are Destroying Good Management Practices, doi: http://dx.doi.org/10.5465/AMLE.2005.16132558, Academy of Management Learning \& Education, 4 (1), 75-91 (2005)

Gómez, M., E. Aranda y J. Santos, A Competency Model for Higher Education: an assessment based on placements, doi: 10.1080/03075079.2016.1138937, Studies in Higher Education, 42 (12), 2195 - 2215 (2017)

Gupta, P.B., P. Saunders y J. Smith, Traditional master of business administration (MBA) versus the MBA with specialization: A disconnection between what business schools offer and what employers seek, doi:10.3200/JOEB.82.6.307-312, Journal of Education for Business, 82(6), 307-312 (2007)

Mintzberg, H. y J. Gosling, Reality Programming for MBAs, ISSN: 1083-706X, Strategy and Business, 26 (1), 28-31 (2002)

Muñoz, R., El Problema de las Maestrías en Administración en Colombia: una Propuesta Curricular, doi: https://doi.org/10.25100/cdea.v20i32.206, Cuadernos de Administración, 32, 137-150 (2004)

Olivencia, J.J.L., E.P. García y R.P. Galán, Las Comunidades de Aprendizaje como Estrategia de Desarrollo y Compromiso Pedagógico en la Formación Inicial del Profesorado, ISSN-e 1989-3558, Revista Educativa Digital, (16) 51$58(2014)$

Ortega, J.L., Redes Sociales Académicas ¿Espacios Colaborativos o Clubes Diógenes?, doi: https://doi.org/10.3145/thinkepi.2017.42, Anuario ThinkEPI, (1), 225-229 (2017)

Punie, Y., Learning Spaces: An ICT- Enabled Model of Future Learning in the Knowledge - Based Society, doi:10.1111/j.1465-3435.2007.00302.x/, European Journal of Education, 42 (2), 185 - 199 (2007)

Rodríguez, M. y F. Hinojo, Incidencia de los Programas de Formación Pedagógica en el Perfil de los Profesionales No Licenciados en la Facultad de Educación de UNIMINUTO, doi: 10.4067/S0718-50062017000500003, Formación Universitaria, 10(5), 17-28 (2017)

Rubin, R. S., y E. C. Dierdorff, Building a better MBA: From a decade of critique toward a decennium of creation, doi. https://doi.org/10.5465/amle.2012.0217, Academy of Management Learning \& Education, 12, 125-141 (2013)

Salas, S., Evolution of the Framework for 21st Century Competencies, ISNN: 2073-7904, Knowledge Management \& ELearning: An International Journal, 5 (1), 10 -24 (2013)

Schwab, K., La Cuarta Revolución Industrial, 149. Editorial Debate, Davos, Suiza (2016)

Sistema Nacional de Información de la Educación Superior SNIES, Búsqueda de Instituciones de Educación Superior Aprobadas, M. d. Nacional, Productor (2017)

Tan, B.S. y S. Ko, How relevant is the MBA: A revisit. doi:10.1080/08832323.2018.150358, Journal of Education for Business, 94(1), 64-69 (2019)

Yáñez, G.L. y B.K. Soria, Reflexión de Buenas Prácticas Docentes como eje de Calidad en la Educación Universitaria: Caso Escuela de Ciencias Empresariales de la Universidad Católica del Norte, doi: 10.4067/S0718-50062017000500007, Formación Universitaria, 10(5), 59-68 (2017) 
